\title{
The role of temperature fluctuations in the dynamics of type-I and type-II edge localized modes at ASDEX Upgrade
}

\author{
IGJ Classen ${ }^{1}$, JE Boom ${ }^{1,2}$, AV Bogomolov ${ }^{1}$, E Wolfrum², M Maraschek ${ }^{2}$, \\ W Suttrop ${ }^{2}$, PC de Vries' ${ }^{1}$, AJH Donné ${ }^{1,3}$, BJ Tobias ${ }^{4}$, CW Domier ${ }^{5}$, NC \\ Luhmann $\mathrm{Jr}^{5}$ and the ASDEX Upgrade Team
}

${ }^{1}$ FOM Institute DIFFER - Dutch Institute for Fundamental Energy Research, Association EURATOM-FOM, 3430 BE Nieuwegein, The Netherlands

${ }^{2}$ Max-Planck-Institut für Plasmaphysik, EURATOM Association, Boltzmannstraße 2, 85748 Garching, Germany

${ }^{3}$ Eindhoven University of Technology, $5600 \mathrm{MB}$ Eindhoven, The Netherlands

${ }^{4}$ Princeton Plasma Physics Laboratory, Princeton, NJ 08540, USA

${ }^{5}$ University of California at Davis, Davis, CA 95616, USA

E-mail:I.G.J.Classen@differ.nl

\begin{abstract}
D temperature measurements using the electron cyclotron emission imaging diagnostic at ASDEX Upgrade revealed a variety of temperature fluctuations associated with type-I and type-II edge localized modes (ELMs). The characteristics and dynamics of these modes, and their role in the ELM cycle, are presented. During type-I ELMs, different phases of distinct mode activity have been identified. At the onset of the ELM crash, a short lived mode is observed in the pedestal region. During the actual crash phase, multiple filamentary structures are observed just outside the separatrix. A third type of fluctuation, the inter-ELM mode, is often observed in between type-I crashes. The occurrence of this mode tends to lengthen the ELM period. During type-I ELM suppression with magnetic perturbation coils, smaller crash events become more frequent, replacing the large type-I crashes. In type-II ELMs, temperature crashes are absent altogether, and a continuous broadband fluctuation in the $20-60 \mathrm{kHz}$ range, showing beat-wave like behaviour, is observed. The similarities between the characteristics of this mode and the inter-ELM mode suggest that it is the same instability. In type-I ELMs it delays the next crash, in type-II ELMs it might be responsible for the complete absence of crash events.
\end{abstract}

\section{Introduction}

Edge localized modes (ELMs) [1] are periodic relaxations of the edge transport barrier (pedestal) in $\mathrm{H}$ mode plasmas [2]. The heat and particle load expelled from the plasma during these crashes could prove difficult to handle, possibly unacceptably shortening the life time of plasma facing components in future fusion reactors [3]. Controlling or mitigating the ELMs is therefore required for the reliable operation of future devices. For the development of control tools (e.g. [4]) it is crucial to understand the physics and dynamics of ELMs. Both type-I and type-II ELMs show magneto-hydrodynamic mode activity. Precursor modes [5,6,7] and filaments [8,9] associated with type-I ELMs have been reported by various devices. Type-II ELMs $[10,11]$ are characterized by a continuous broadband edge fluctuation [12]. Recently, measurements using the electron cyclotron emission imaging (ECEI) diagnostic [13] on ASDEX Upgrade revealed the 2D dynamics of edge electron temperature fluctuations in both type-I [14] and type-II [15] ELMs. This paper gives an overview of the properties 
and dynamics of the various edge temperature fluctuations observed on ASDEX Upgrade. The role of the various fluctuations in the ELM cycle and in the transition between the various ELM regimes (type-I ELMs, type-II ELMs and ELMs mitigated by magnetic perturbations) is investigated.

The ECEI diagnostic provides a localized, high resolution 2D measurement of the electron temperature and its dynamics in a 2D array of 8 (horizontal) by 16 (vertical) positions (128 channels total) in the poloidal plane, covering an area of about $10 \mathrm{by} 40 \mathrm{~cm}$ with a sampling rate of $200 \mathrm{kHz}$. The 2D measurement area is located at the low field side plasma edge, vertically centred on the plasma mid-plane and horizontally (radially) centred on the separatrix.

\section{Type-I ELMs}

Various types of temperature fluctuations have been observed by ECEI during type-I ELM cycles [14]. Figure 1 gives sequences of 2D images of three types of temperature fluctuations measured during a typical ELM cycle (discharge parameters: $B_{t}=2.5 \mathrm{~T}, I_{p}=1 \mathrm{MA}, P_{N B I}=7.5 \mathrm{MW}, P_{E C R H}=0.75 \mathrm{MW}$ ). Just prior to the crash, the ELM onset mode (1) is observed. During the actual ELM crash (the phase in which the pedestal top temperature decreases, lasting about $2 \mathrm{~ms}$ ) multiple filaments (2) rotate past the ECEI viewing window. Often, in between ELM crashes another mode, the inter-ELM mode, (3) occurs. The properties of these modes are described in the following paragraphs. The radiation temperature $T_{r a d}$ is equal to the electron temperature $T_{e}$ only if the plasma is optically thick. The data inside the separatrix (white dashed line) can be considered optically thick (all the red, yellow and white data), see ref [13]. The blue (and black) regions outside the separatrix are optically thin $\left(T_{\text {rad }}\right.$ $<T_{e}$ ).

The poloidal dynamics of the modes often becomes more evident if the resolution in the horizontal direction (i.e. to good approximation the radial direction) of ECEI is sacrificed, and the average over the horizontal lines of sight is plotted as a function of time and vertical position. Each line of sight (LOS) consists of 8 channels, ranging from $R=2.09-2.19 \mathrm{~m}$ for the data shown in this paper. Figure 2 shows LOS averaged ECEI signals (for both the inner and outer four channels of each LOS), along with the divertor shunt current (current flowing between the plasma and the plasma facing components, used as an ELM indicator) for three subsequent ELM crashes (discharge parameters: $\left.B_{t}=2.5 \mathrm{~T}, I_{p}=1 \mathrm{MA}, P_{N B I}=7.5 \mathrm{MW}, P_{E C R H}=1.5 \mathrm{MW}\right)$. The LOS averaged signals are normalized to the average temperature over a full ELM cycle.
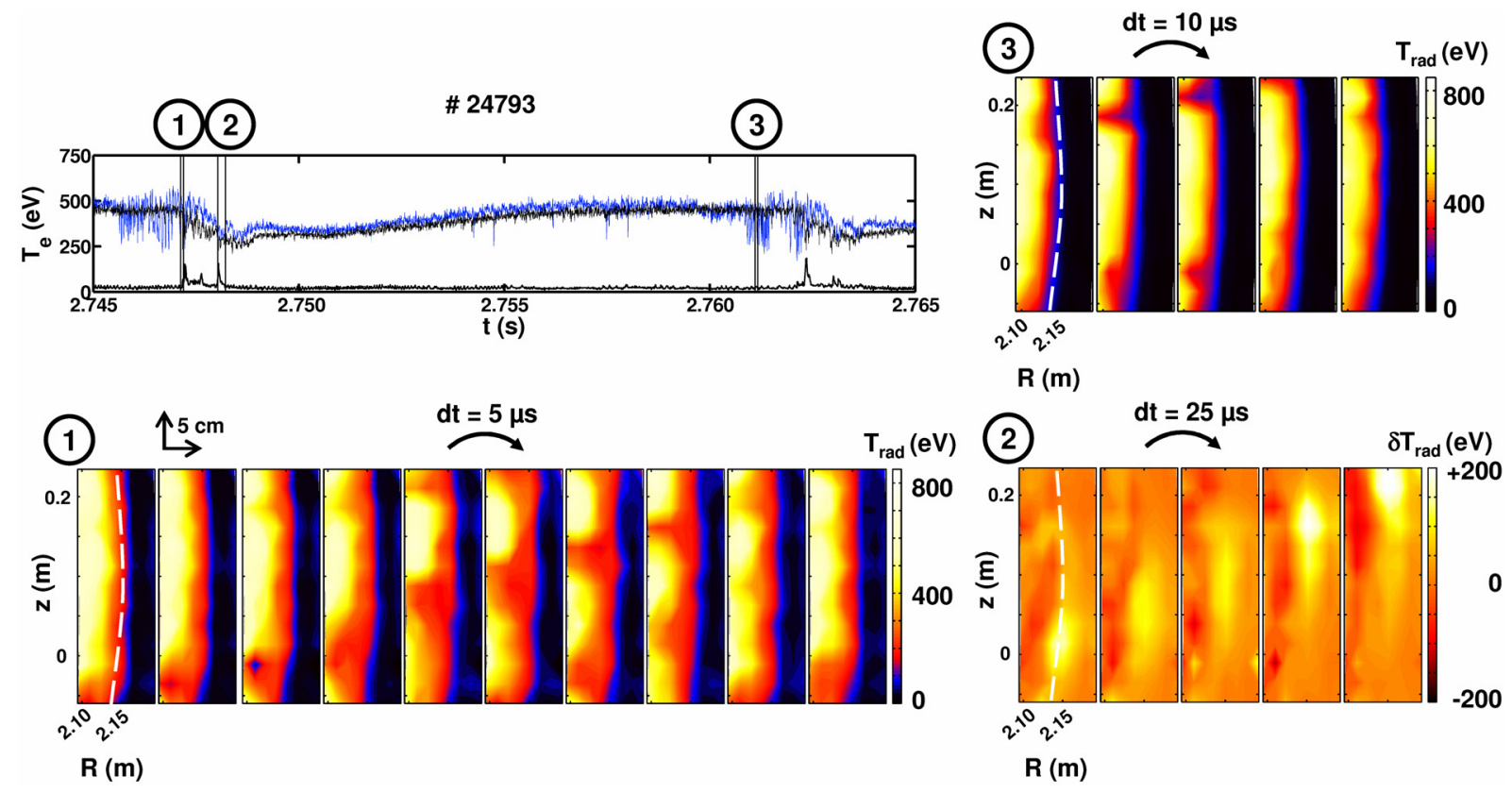
Figure 1. 2D image sequences of the various phases of temperature fluctuations observed during a typical type-I ELM cycle. The upper left plot gives the temperature time traces of three ECEI channels (upper black: pedestal top on axis, blue: pedestal top off-axis, lower black: outside separatrix) and indicates the timing of the three modes within the ELM cycle. The detailed 2D temperature evolution of the three modes is given in the lower left, lower right and upper right plots respectively. The white dashed line in each plot indicates the position of the separatrix (from equilibrium reconstruction). The onset mode (1) leads to the actual crash. During the crash phase, filaments (2) are observed. In between two ELM crashes, often the inter-ELM mode (3) is observed, delaying the next crash.

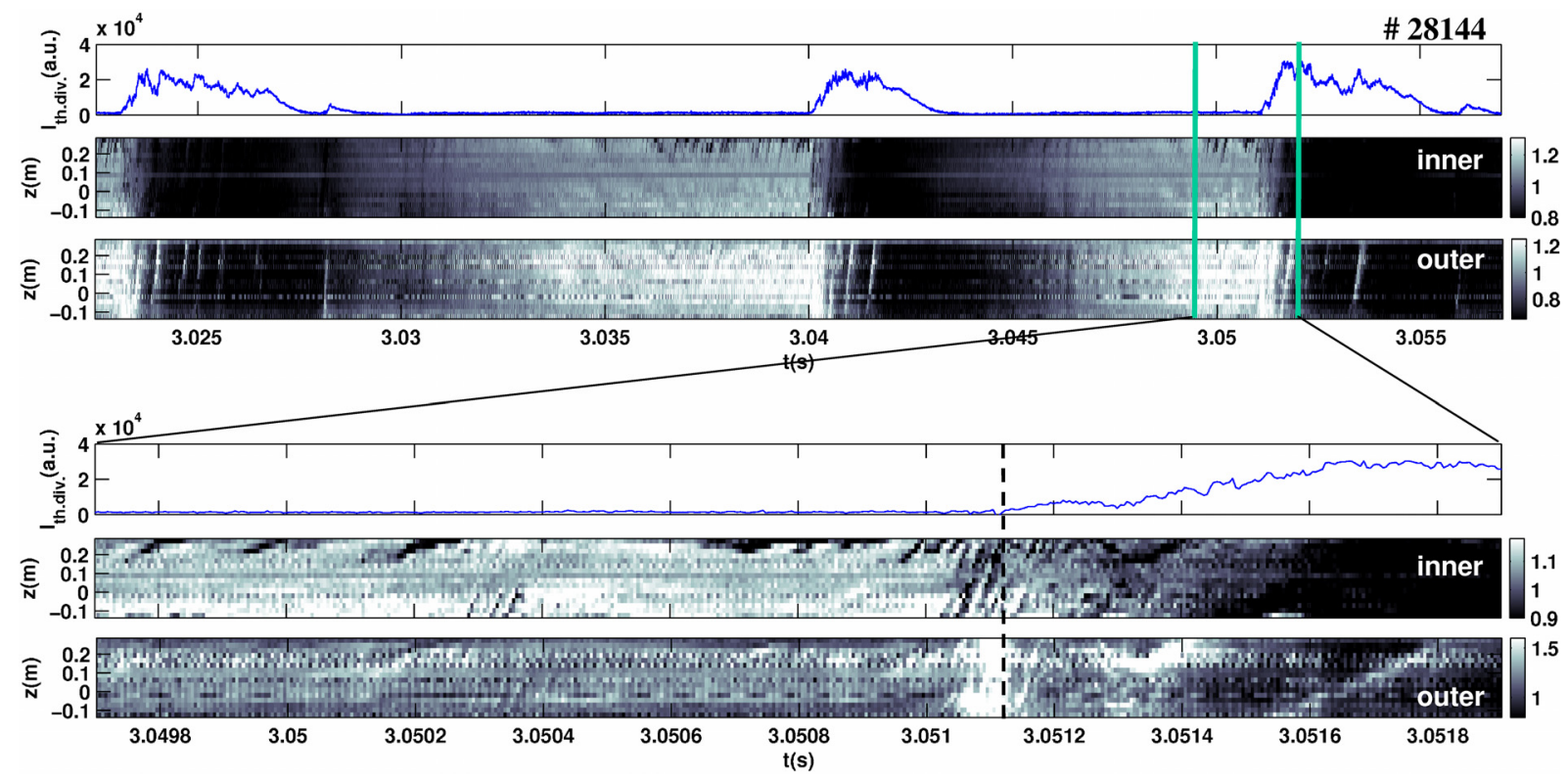

Figure. 2. Type-I ELM fluctuation overview. The top 3 plots give the divertor shunt current and LOS averaged ECEI signals (for both the inner and outer half of the channels) for three subsequent ELM crashes. The outer channels clearly show upward moving filaments during the crash phase which are roughly correlated with the fine structure in the divertor current. In the bottom three plots a zoom on one of the crashes (dotted line indicates the beginning of the crash) is given, showing the onset mode (3.051s-3.0512s), the first clear filament (starting at 3.0516s) and off-axis fluctuations before the crash.

\subsection{Onset mode}

Prior to the onset of the ELM crash, a short lived mode (lasting only a few mode periods) is observed in the pedestal region, shown in both figure 1 (mode 1) and figure 2 as an upward moving undulation in the temperature pedestal with a typical radial displacement of $4 \mathrm{~cm}$ and a poloidal wavelength of about $20 \mathrm{~cm}$. The mode rotates in the electron diamagnetic drift direction at a velocity of $4-10 \mathrm{~km} / \mathrm{s}$. Often (but not always), an increase in poloidal velocity is observed. Only after this mode, the temperature starts to drop and the divertor shunt current signal rises, indicating this mode triggers the actual ELM crash.

\subsection{Crash phase and filaments}

Initially, incoherent and chaotic temperature fluctuations are often seen during the early phase of the crash. As the crash develops, multiple filamentary structures are observed just outside the separatrix, one of them is shown in figure 1 (mode 2). To make the filament more visible, the background temperature profile (taken just after the filament has passed) has been subtracted, and only the temperature difference $\delta T_{\text {rad }}$ is plotted. The filaments are about $200 \mathrm{eV}$ hotter than the surrounding plasma, have a poloidal size of $5-10 \mathrm{~cm}$, and a radial size of about $3 \mathrm{~cm}$. Note that the data shown in figure 1 (mode 2) is partly optically thin (outside the separatrix), so the stated $200 \mathrm{eV}$ should be considered an underestimation (as $T_{r a d}<T_{e}$ for optically thin plasmas). Caution is also needed in the 
exact determination of the position of the filaments, as the effect of 'shine-through' (anomalous ECE radiation outside of the separatrix, described in detail in $[16,17])$ could have affected the data. The radial layer affected by shine-through is however expected to be very narrow for these discharges.

In figure 2, three ELM crashes are shown, where the filaments are visible in the LOS averaged signals of the outer ECEI channels. The filaments occur during the phases of elevated divertor shunt current. The fine-structure in the inner divertor shunt current spikes (which is a toroidally localized measurement $5 / 16^{\text {th }}$ of a full torus away from ECEI), shows a rough correlation with the filaments observed by ECEI. Most of these filaments are observed to rotate in the electron diamagnetic drift direction with a velocity of about $2 \mathrm{~km} / \mathrm{s}$, although sometimes the first filament is seen to move in the opposite direction (e.g. at $t=3.0513 \mathrm{~s}$ where a filament reverses rotation).

\subsection{Inter-ELM mode}

A third type of fluctuation is often observed in between ELM crashes, shown in figure 1 (mode 3) as upward moving dips in the temperature pedestal with a typical radial displacement of $3 \mathrm{~cm}$. This mode rotates in the electron diamagnetic drift direction, has a poloidal wavelength of about $10 \mathrm{~cm}$ and a frequency in the $20-50 \mathrm{kHz}$ range. The velocity of this mode can vary significantly, but is typically $2-$ $4 \mathrm{~km} / \mathrm{s}$. It shows a pronounced poloidal amplitude asymmetry, with a clear amplitude minimum on the plasma mid-plane. The upward rotating temperature dips significantly decrease in amplitude or completely disappear when traversing the mid-plane. Often dips seem to originate just above the midplane or terminate just below it (as in figure 1, mode 3). Not all ELM cycles show inter-ELM modes (e.g. the ELMs in figure 2 do not), and if the mode is present its duration varies significantly (from $0.1 \mathrm{~ms}$ to almost the entire inter-ELM period). Figure 4 (case 1) shows an example of a type-I ELM crash with strong inter-ELM mode activity, also clearly illustrating the amplitude minimum on the plasma mid-plane (the mode is only clearly visible above and below the mid-plane). Often, two distinct ELM periods are observed in ASDEX Upgrade discharges [18]. Only the 'slow' ELMs show inter-ELM mode activity. So, the occurrence of this mode seems to delay the next type-I ELM crash, suggesting it regulates the stability of the pedestal.

\subsection{Small intermediate crashes and type-I ELM suppression by magnetic perturbations}

Sometimes small intermediate crashes are observed in between (large) type-I crashes. These small crashes, that only cause a $T_{e}$ drop of at most $10 \%$, are triggered by a short lived (about $0.2 \mathrm{~ms}$ ) mode with a poloidal wavelength around $20 \mathrm{~cm}$, rotating in the electron diamagnetic drift direction at 4$8 \mathrm{~km} / \mathrm{s}$. This mode hence has a lot of similarities with the onset mode. These small intermediate crashes, that delay the next type-I crash, seem to occur more frequently at higher edge densities. They also seem to play an important role in the suppression of type-I ELMs using magnetic perturbation (MP) fields [4]. Figure 3 shows the suppression of type-I ELMs using MP coils (discharge parameters: $\left.B_{t}=2.5 \mathrm{~T}, I_{p}=0.8 \mathrm{MA}, P_{N B I}=7.5 \mathrm{MW}, P_{E C R H}=1.4 \mathrm{MW}\right)$. Above a certain edge density threshold $\left(\sim 6.5 \cdot 10^{19} \mathrm{~m}^{-3}\right)$ the large type-I crashes disappear (are suppressed), and only the much smaller intermediate crashes remain. As the density approaches the threshold, the small crashes become more frequent (up to $500 \mathrm{~Hz}$ ), while the type-I crash frequency (initially $100 \mathrm{~Hz}$ ) decreases. The observation that the small crashes are seen to increase in frequency with density, and that they delay the next typeI ELM, suggests that during MP suppression the increased frequency of the small events might be responsible for the suppression of the type-I crashes. 


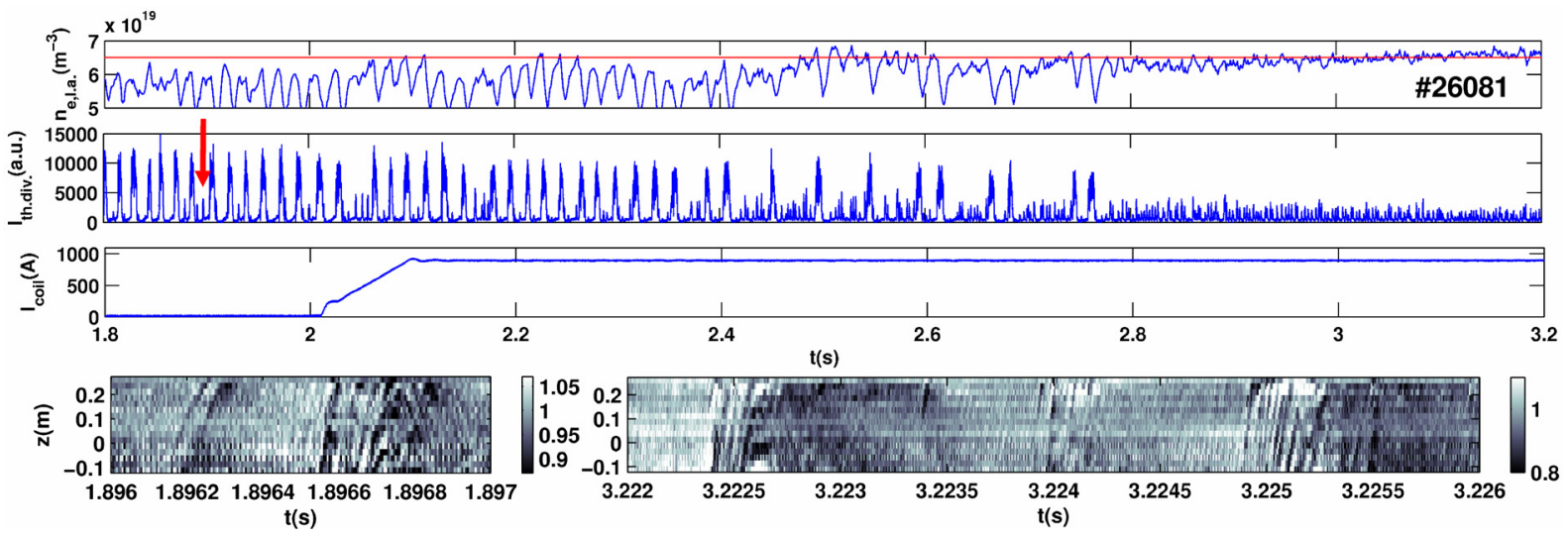

Figure 3. Type-I ELM suppression by MP coils. The top three plots respectively show the line averaged density at the plasma edge (the red line indicating the empirical threshold for MP suppression), the divertor shunt current showing the increasing number of small intermediate crashes in between the large type-I crashes and the MP coil current. At the bottom, the LOS averaged ECEI signals for both an intermediate crash before MP application (left, timing indicated by red arrow in $\left.I_{\text {th.div. }}\right)$ and a few small crash events during MP suppression (right) are shown.

\section{Type-II ELMs}

In figure 4, a transition between type-I and type-II ELMs is shown. This transition is induced by a shift towards a double null configuration in a highly shaped plasma at high edge collisionality $\left(B_{t}=2.5 \mathrm{~T}, I_{p}=0.8 \mathrm{MA}, P_{N B I}=7.5 \mathrm{MW}, P_{E C R H}=0.9 \mathrm{MW}\right.$, see [15] for more parameters). The ELM crashes, as seen in the divertor current, become more frequent and smaller and finally disappear. One of the main characteristics of type-II ELMs is the occurrence of a broadband fluctuation in the $20-60 \mathrm{kHz}$ range [12], measured by magnetics.

The LOS averaged ECEI signals in figure 4 show strong mode activity during this transition. The pure type-I ELMs (figure 4, case 1) already show strong inter-ELM mode activity before the crashes. The $5 \mathrm{~ms}$ time interval shown corresponds to about one third of a complete ELM period. The actual crash phase is indicated by the horizontal arrow (indicating the phase of elevated divertor shunt current) and continues another $2 \mathrm{~ms}$ beyond the bound of the figure. The modes are seen to gradually slow down (starting around $40 \mathrm{kHz}$ ) before the crash. The onset mode is not very pronounced in these crashes, but can be observed as a sudden speeding up of the mode just before the crash phase (at $t=2.46 \mathrm{~s}$ ). Note that the divertor shunt current is already slightly elevated during the presence of the inter-ELM mode, indicating an energy loss due to this mode. After the crash there is a longer period (typically 10ms) with no inter-ELM mode activity (not shown in the figure).

During the transition, the average inter-ELM mode frequency increases towards $40 \mathrm{kHz}$, as the slowing down phase before the crash becomes less pronounced. However, large frequency (poloidal velocity) changes are always present. Although the crashes become more frequent, they also become smaller (shorter crash phases, lower temperature drop) and more and more dominated by the inter-ELM mode. One of the intermediate crash events is shown in figure 4, case 2. The $7 \mathrm{~ms}$ time interval shown corresponds to about one complete crash period. This crash no longer looks like a normal type-I crash. No large temperature drop is observed (the color scaling does not go black after the crash as observed in the pure type-I case), and the inter-ELM mode never completely disappears, but seems to be locking and even reversing its rotation direction during the residual crash event. 


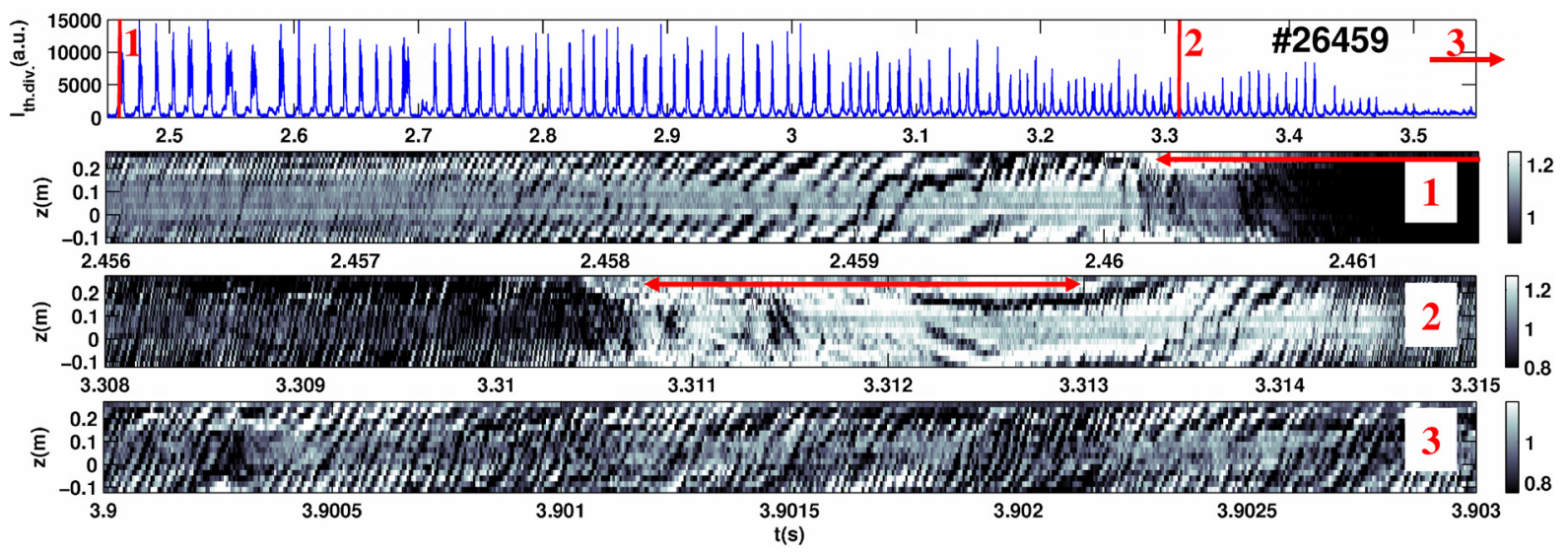

Figure 4. Transition from type-I to type-II ELMs. The divertor shunt current (top) shows that the crashes become faster and smaller (both the duration and height of the current peaks decrease) and finally completely disappear. The LOS averaged ECEI signals for a pure type-I ELM crash (1), an intermediate residual small crash event (2) and a pure type-II phase (3) are shown. The inter-ELM mode already strongly present in the type-I phase becomes more and more dominant over the transition. The horizontal arrows in panels 1 and 2 indicate the phases of elevated divertor shunt current (i.e. the actual crash phases).

In the pure type-II phase (figure 4, case 3 ) no temperature crashes are observed anymore, but a continuous mode with poloidal wavelength around $10 \mathrm{~cm}$, rotating in the electron diamagnetic drift direction at typically $5 \mathrm{~km} / \mathrm{s}$ is observed. This is the same mode as the $20-60 \mathrm{kHz}$ broadband fluctuation measured by magnetics. The broadband character is explained by the significant variation in the observed rotation velocity. This type-II ELM mode is seen to reach a maximum relative temperature amplitude of $20 \%$ just inside the top of the pedestal, while simultaneously flattening the $T_{\mathrm{e}}$ profile at this location [15]. As with the inter-ELM mode in between type-I crashes, also the time averaged 2D mode amplitude of this type-II ELM mode shows an amplitude minimum at the plasma mid-plane. Whether this minimum is real (as partly supported by recent results from the JOREK code, where offaxis behaviour of magnetic quantities is reported [19]), or a diagnostics artefact related to edge density perturbations causing beam refraction is still unresolved (this applies to both the type-II ELM mode and the inter-ELM mode). The large similarities between the inter-ELM mode and the type-II mode (and the smooth transition between them) suggest that these modes are the same. In type-I ELMs it delays the next crash, in type-II ELMs it might be responsible for the complete absence of crash events. The apparent contradiction that, during the type-I to type-II transition, the increased activity of the mode increases the frequency of the crashes (instead of delaying the next type-I crash) is probably due to the changing character of the crashes that can no longer be considered type-I crashes.

A more detailed investigation of the type-II mode reveals phases of high and low mode amplitude that are very reminiscent of beat-wave patterns in both space and time. In figure 5, LOS averaged ECEI signals for the type-II mode are plotted both as a contour plot and as stacked time traces. The data has been high pass filtered to suppress slower drifts in the amplitude and to only show the fast oscillation of interest. Especially in the stacked time trace plot the phases of high and low mode amplitude are clearly visible. The bands of high amplitude are seen to propagate in the same direction as the individual modes, but approximately ten times slower. If these patterns are indeed due to beat-waves, the type-II mode actually consists of multiple simultaneous modes (with different mode number and frequency) that are alternately interfering destructively and constructively. The beat-wave frequency in time (which is the frequency difference between the beating waves) is roughly $1 \mathrm{kHz}$. The beat-wave period in space is in the order of $40 \mathrm{~cm}$.

These beat-wave patterns can alternatively be interpreted as a strong poloidal localization of the mode, possibly explaining the observed poloidal asymmetries in the time averaged 2D mode amplitude. The observed pronounced minimum at the mid-plane however only becomes apparent when averaging over a longer time interval. There are phases (e.g. at 4.1207s) where the mode amplitude even has its 
maximum at the mid-plane. Why however, averaged over time, the minimum seems to be predominantly located at the mid-plane is still unresolved.

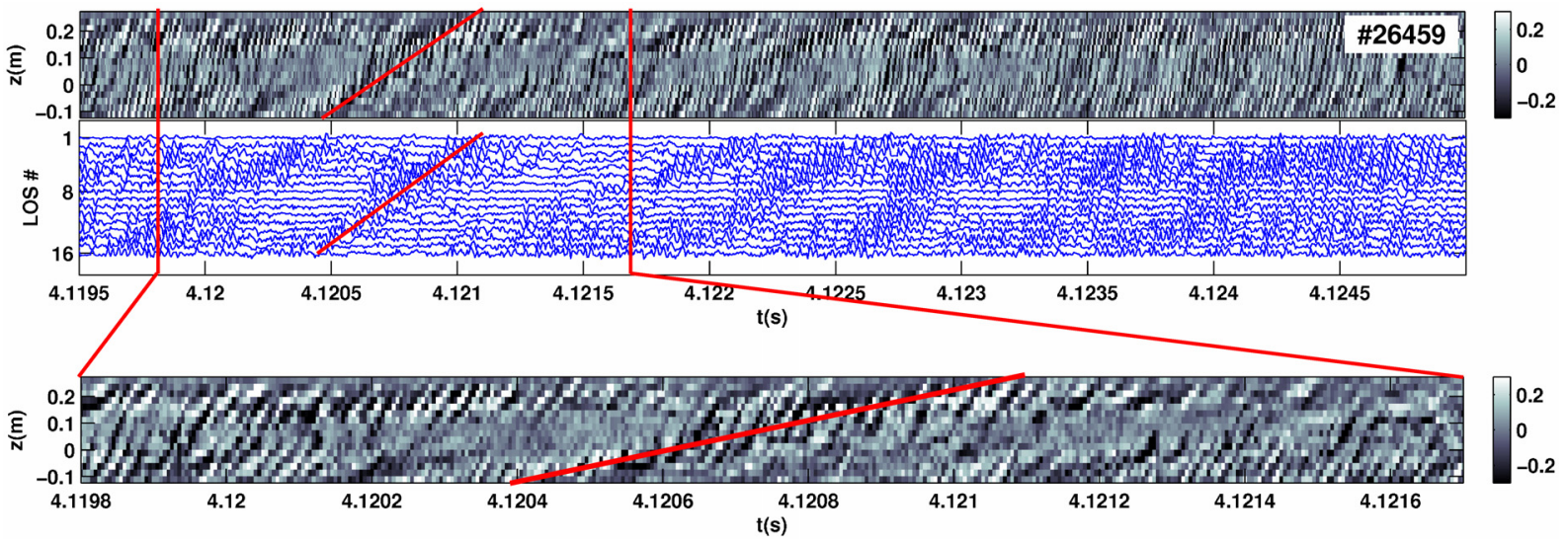

Figure 5. The top two figures show identical LOS averaged ECEI signals plotted once as a contour plot and once as stacked time traces. Phases of high and low mode amplitude, traveling across the ECEI viewing window at a velocity much slower than the individual mode velocity, can be observed (especially in the stacked time trace plot), very reminiscent of beat wave patterns. The zoom at the bottom shows one of the poloidally highly localized high amplitude phases passing through the ECEI viewing window.

\section{Rotation of the fluctuations}

The observed rotation of the modes is predominantly in the electron diamagnetic drift direction. The modes are expected to rotate with the background plasma at the $E \times B$ drift velocity $v_{E \times B}=-E_{r} / B$ [20] and possibly with an additional (diamagnetic) mode propagation velocity. In H-mode, the profile of the radial electric field $E_{r}$ shows a negative well at the plasma edge $(\rho>0.95)$, with a minimum close to the separatrix [21]. At the plasma edge this indeed results in a (poloidal component of the) $E \times B$ drift velocity in the electron diamagnetic drift direction ('up' on the LFS), explaining the rotation direction of the modes.

The onset mode is the fastest rotating mode with a velocity of $4-10 \mathrm{~km} / \mathrm{s}$. The inter-ELM mode rotates at roughly half that speed $(2-4 \mathrm{~km} / \mathrm{s})$, similar to the type-II modes $(5 \mathrm{~km} / \mathrm{s})$. All these velocities are below the maximum $E \times B$ drift velocity in the minimum of the $E_{r}$ well (roughly $15 \mathrm{~km} / \mathrm{s}$ for $7.5 \mathrm{MW}$ NBI heated plasmas). The filament rotation velocity is typically slower than the other modes, around $2 \mathrm{~km} / \mathrm{s}$, and increases during the crash phase. This could be explained by the collapse and subsequent recovery of the $E_{r}$ well during a type-I crash [21]. In the early phases of the crash, the rotation velocity of the filaments is even occasionally seen to reverse, possibly indicating that a temporary $E_{r}$ inversion occurs.

The depth of the $E_{r}$ well generally increases with heating power. However, no clear correlation between the rotation velocity of the modes and the heating power has been observed, possibly indicating that the modes are not associated with the minimum in $E_{r}$, but are positioned more towards the pedestal top (where $E_{r}$ is smaller but still negative). Also, within a single ELM period, the modes (often abruptly) significantly change their rotation velocity or even reverse.

Note that the radial width of the modes (several $\mathrm{cm}$ ) is larger than the width of the $E_{r}$ well. Nevertheless, the modes rotate with a single rotation velocity. Hence, no velocity shearing of the modes is observed; data from different radii show the same mode velocity. 


\section{Discussion and Conclusions}

All observed modes, except the filaments, show similar properties. They are all located near the pedestal top, rotate in the electron diamagnetic drift direction $\left(E \times B\right.$ drift in the $E_{r}$ well) with velocities up to $10 \mathrm{~km} / \mathrm{s}$ and are all (to a certain extent) coherent oscillations with poloidal wavelength between $10-20 \mathrm{~cm}$, corresponding to poloidal mode numbers in the order of $m=60-120$ (when assuming the modes to be located at $\rho=0.95$ and taking straight field line angle corrections into account) and toroidal mode numbers $n=m / q_{95}=10-25$ ( $q_{95}$ is the safety factor at $\rho=0.95$ ). Often a poloidal localization is observed. These properties are in the right ballpark as expected for (peeling-)ballooning modes, which are the most likely theoretical candidate for explaining the (linear onset of the) ELM crash [22]. Note that the long poloidal wavelength modes $(20 \mathrm{~cm}$ for onset-mode and intermediate crash mode) result in crashes, whereas the short poloidal wavelength modes $(10 \mathrm{~cm}$ for inter-ELM and type-II mode) do not, in line with the prediction that higher $m$ ballooning modes are radially more localized and hence have a smaller influence on the confinement [23]. Filaments are positioned further outside (at or outside the separatrix) and are observed as highly localized single blobs. In recent nonlinear simulations with the reduced MHD code JOREK [19], similar high poloidal localization, resulting from mode coupling of multiple ballooning modes, was obtained. The experimental observations of all the modes, including filaments, are hence not incompatible with the prevailing peeling-ballooning model, but do illustrate that the dynamics is more diverse and complicated. Especially the rapidly varying rotation of the modes (including rotation reversals) is still poorly understood.

Both in the case of type-II ELMs and type-I ELM suppression by magnetic perturbations, a less violent instability becomes dominant, preventing the more violent type-I crashes to occur. During suppression of type-I ELMs using magnetic perturbation fields, it is observed that small crash-like events become more frequent as the density increases, and finally completely replace the large type-I crashes. These small crash events are already sporadically present without perturbation fields (and at low density), and are observed to delay the next large type-I crash (hence resulting in a more stable pedestal). Similarly, the inter-ELM mode is observed to delay the next type-I crash. During the transition from type-I to type-II ELMs, this mode becomes more and more dominant, finally completely preventing the type-I crashes.

This paper aims at giving a description of the 'typical' ELM dynamics. It should however be noted that a significant variation of the ELM dynamics is observed when analyzing large number of ELMs, even within the same discharge with the same background plasma parameters. Hence, in individual ELMs, significant deviations from the mentioned dynamics, velocities, mode numbers, amplitudes and frequencies are to be expected.

Acknowledgements - This work, supported by the European Communities under the Contract of Association between EURATOM-FOM, was carried out within the framework of the European Fusion Program, with financial support from NWO, FOM and EURATOM. The views and opinions expressed herein do not necessarily reflect those of the European Commission.

\section{References}

[1] Zohm H, "Edge localized modes (ELMs)", Plasma Phys. Control. Fusion 38 (1996) 105

[2] Wagner F, et al., "Regime of Improved Confinement and High Beta in Neutral-Beam-Heated Divertor Discharges of the ASDEX Tokamak" Phys. Rev. Lett. 49 (1982) 1408

[3] Loarte A, et al., "Characteristics of type I ELM energy and particle losses in existing devices and their extrapolation to ITER" Plasma Phys. Control. Fusion 45 (2003) 1549

[4] Suttrop W, et al., "First Observation of Edge Localized Modes Mitigation with Resonant and Nonresonant Magnetic Perturbations in ASDEX Upgrade", Phys. Rev. Lett. 106 (2011) 225004

[5] Oyama N, et al., "Fast dynamics of type I ELMs and transport of the ELM pulse in JT-60U" Nucl. Fusion 44 (2004) 582

[6] Terry JL, et al., "The dynamics and structure of edge-localized-modes in Alcator C-Mod" $J$. Nucl. Mater. 363-365 (2007) 994 
[7] Oyama N, et al., "Characteristics and control of the type I edge localized mode in JT-60U" Nucl. Fusion 51 (2011) 033009

[8] Kirk A, et al., "Spatial and Temporal Structure of Edge-Localized Modes" Phys. Rev. Lett. 92 (2004) 245002

[9] Eich T, et al., "Nonaxisymmetric Energy Deposition Pattern on ASDEX Upgrade Divertor Target Plates during Type-I Edge-Localized Modes" Phys. Rev. Lett. 91 (2003) 195003

[10] Ozeki T, et al., "Plasma shaping, edge ballooning stability and ELM behaviour in DIII-D" Nucl. Fusion 30 (1990) 1425

[11] Stober J, et al., "Type II ELMy H modes on ASDEX Upgrade with good confinement at high density" Nucl. Fusion 41 (2001) 1123

[12] Wolfrum E, et al., "Characterization of edge profiles and fluctuations in discharges with type-II and nitrogen-mitigated edge localized modes in ASDEX Upgrade", Plasma Phys. Control. Fusion 53 (2011) 085026

[13] Classen IGJ, et al., "2D electron cyclotron emission imaging at ASDEX Upgrade", Rev. Sci. Instrum. 81 (2010) 10D929.

[14] Boom JE, et al., "2D ECE measurements of type-I edge localized modes at ASDEX Upgrade", Nucl. Fusion 51 (2011) 103039

[15] Boom JE, et al., "Characterization of broadband MHD fluctuations during type-II edge localized modes as measured in 2D with ECE-imaging at ASDEX Upgrade", Nucl. Fusion 52 (2012) 114004

[16] Tobias BJ, et al., "ECE-imaging of the H-mode pedestal", Rev. of Sci. Instrum. 83 (2012) $10 \mathrm{E} 329$.

[17] Rathgeber S, et al., "Estimation of edge electron temperature profiles via forward modelling of the electron cyclotron radiation transport at ASDEX Upgrade", Plasma Phys. and Control. Fusion 55 (2012) 025004

[18] Burckhart A, et al., "Inter-ELM behaviour of the electron density and temperature pedestal in ASDEX Upgrade", Plasma Phys. Control. Fusion 52 (2010) 105010

[19] Hölzl M, et al., "Reduced-magnetohydrodynamic simulations of toroidally and poloidally localized edge localized modes", Phys. Plasmas 19 (2012) 082505

[20] Stroth U, et al., "On the interaction of turbulence and flows in toroidal plasmas", Plasma Phys. Control. Fusion 53 (2011) 024006

[21] Viezzer E, et al., "High-accuracy characterization of the edge radial electric field at ASDEX Upgrade", submitted to Nucl. Fusion

[22] Snyder PB, et al., "Edge localized modes and the pedestal: A model based on coupled peelingballooning modes", Phys. Plasmas 9 (2002) 2037

[23] Wilson HR, et al., "Numerical studies of edge localized instabilities in tokamaks", Phys. Plasmas 9 (2002) 1277 